\title{
Fungating Wounds - Multidimensional Challenge in Palliative Care
}

\author{
Thomas Merz $^{\mathrm{a}}$ Carsten Klein ${ }^{\mathrm{a}}$ Barbara Uebach ${ }^{\mathrm{b}}$ Martina Kern ${ }^{\mathrm{b}}$ \\ Christoph Ostgathe ${ }^{\mathrm{a}}$ Johannes Bükki ${ }^{\mathrm{a}}$ \\ aDivision of Palliative Medicine, University of Erlangen, \\ ${ }^{b}$ Centre for Palliative Medicine, Malteser Krankenhaus Bonn, University of Bonn, Germany
}

\section{Keywords}

Local wound management - Odour . Pain · Bleeding . Necrosis

\section{Summary}

The management of fungating, malignant wounds is a challenge for the palliative care team. Open, malodorous, poorly healing lesions are obvious signs of underlying disease. In addition, pain and functional impairment remind the patients of their incurable illness. A multidimensional approach is necessary to meet the needs of these patients and to improve quality of life. Although achieving wound closure is rarely a realistic goal, modern techniques of wound management can help to minimize odours and exudates. Specialist knowledge in palliative care is needed to provide adequate pain control. Psychosocial support may help patients to cope with the situation and their limited abilities.

\section{Introduction}

Locally advanced, metastatic or recurrent cancer may infiltrate the skin, disrupt its integrity, and cause chronic, poorly healing, and fungating wounds. Closure of these wounds is difficult and in many cases impossible, which causes significant distress for patients, families and the caregiving teams. Moreover, they are a constant and perceptible reminder (vision, smell, discharge) of disease progression. Frequent complications of these wounds are bleeding, infections, and sepsis. The patients suffer from pain, functional impairment, the presence of an open, discharging wound, and very often from odours due to necrosis and microbial contamination. Palliative care directly addresses the needs of the patients and their

\author{
Schlüsselwörter \\ Lokale Wundversorgung · Geruchsbildung · Schmerz · \\ Blutung · Nekrosen
}

\section{Zusammenfassung}

Maligne, exulzerierende Wunden sind ein sichtbares Zeichen fortgeschrittener Erkrankung und führen nicht nur zu körperlichen Symptomen sondern können auch soziales, psychisches und existentielles Leiden verursachen. Diesem Leiden auf unterschiedlichen Ebenen zu begegnen, erfordert eine multidimensionale Herangehensweise. Auch wenn ein Wundverschluss selten erreicht werden kann, so sind Maßnahmen der modernen Wundversorgung doch in der Lage, Gerüche zu binden und Sekretion zu reduzieren. Zu einer guten Wundversorgung gehört auch die kompetente Behandlung von Schmerzen. Unterstützung im psychosozialen und spirituellen Bereich kann Patienten und Angehörigen helfen, sich mit den Veränderungen auseinanderzusetzen.

relatives in such distressing situations. Principles of care are to be defined according to the goals of palliative care in general. Primarily the underlying problem has to be addressed aiming at a best possible wound management or disease control. This is done by an interdisciplinary approach integrating local or systemic modalities such as surgery, chemotherapy, hormonal manipulation, and radiotherapy. If cure is not achievable or the oncologic armamentarium is not appropriate, the primary goal is shifted towards providing the best comfort and quality of life possible despite the presence of the ulcerating wounds. Thus, local wound management and symptom control become the major focus of the multidimensional approach integrating physical, psychological, social, and spiritual issues.

\section{KARGER \\ Fax +497614520714 \\ Information@Karger.de}

www.karger.com (c) 2011 S. Karger GmbH, Freiburg

$1661-3791 / 11 / 0061-0021 \$ 38.00 / 0$

Accessible online at:

www.karger.com/brc
Thomas Merz

Palliativstation

Universitätsklinikum Erlangen

Krankenhausstrasse 12, 91054 Erlangen, Germany

Tel. +49 9131 853406-4, Fax -6

thomas.merz@uk-erlangen.de 
Table 1. Individual assessment and documentation of fungating wounds

Patient data (demographics, diagnosis, co-morbidities)
Size, type, and location of the wound
Extent
Depth/height
Wound characteristics
Moisture
Bleeding
Secretion
Colour
Odour
Pain
In general
At the wound
During change of dressing
Other symptoms connected with the wound (e.g. itching)
Patients wishes
Aims of wound care
With respect to the wound itself
With respect to quality of life
Care used for the wound
Cleaning
Irrigation
Measures for odour reduction
Dressing
Agreement of the patient to having photographs included in the
documentation
Photo-documentation
Pes of carrying out the care
Paritien

\section{Local Wound Management}

The procedures appropriate for local wound management are determined by the individual size, shape, and location of the ulcerating tumour, the predominant symptoms, and the needs of the patient and his next of kin. In order to cope with the complexity of the situation, careful assessment and continuous documentation (table 1 ) is necessary.

\section{Wound Dressing}

Wound dressings can have a significant impact on the patients' comfort. They may relieve pain, ameliorate odours, reduce bleeding, and absorb exudates. Above that, an appropriate wound dressing may facilitate patients to be socially active by concealing disfigurement.

Dressings for discharging wounds should be as thin as possible to be comfortable. They have to support mobility and therefore be adapted to anatomical conditions. If dressings are too voluminous, pressure can lead to discomfort, for example in the axillary region. The dressing sticking to the wound is a common problem. Therefore, the wound surface must be kept moist in order to prevent adherence, for example by using materials with non-adherent properties. Moreover, the dressings need to have a high ability to absorb and store fluids on the one hand and a high capacity to evaporate these fluids on the other hand, in order to extract as much water content as possible from the wound. Exudates have to be absorbed to prevent further maceration of the surrounding tissue; additionally, covering ointment may be applied. The effect is directly associated with the dressing's fitting to the size and shape of the wound. The integrity of the surrounding skin can be preserved by using the appropriate configuration and materials for adhesives [1].

Silver-impregnated wound dressings help to reduce microbial growth and therefore odours. A very efficient way to bind secretions and odours is the use of activated charcoal. Crushed tablets of activated charcoal (as used for diarrhoea treatment) can be packed into compresses or other appropriate tissues and applied to the top layer of the dressing. Direct contamination of the wound with the charcoal has to be avoided. Commercially available products provide a more refined but at the same time more expensive alternative.

Pain management should follow the WHO guidelines [2] with regular and an on-demand analgesic medication especially in cases of painful dressing changes. The dressing has to be applied and later removed as carefully as possible to avoid pain and unnecessary distress. The application intervals shall be as short as necessary and as long as possible. If changing the dressing is painful and on-demand medication is not sufficient, analgesia combined with mild sedation to facilitate the procedure and to reduce distress may be appropriate. The role of topical opioids in ulcerating cancer lesions is still unclear.

\section{Local Care-Odour}

Another important goal of symptom-oriented care in the case of fungating wounds is the treatment of odours which are often caused by infections. Topical application of antibiotics that reduce the number of the mainly anaerobic microorganisms (e.g. metronidazole) may reduce local infection and thereby ameliorate bothersome smells. Additional systemic medication, when sensitivity in culture is proven, may be beneficial. Treatment of infection may itself have an additional positive impact on the amount of exudate. The local use of disinfecting solutions (e.g. phenoxyethanol 2\%) may be additionally supportive. Symptomatically, odours can be reduced by topical application of chlorophyll. A disadvantage is the green discoloration of the wound. Orally administered chlorophyll may be equally effective. Medical honey, applied as a dressing in fungating wounds, is known to have odourreducing properties [3].

If irrigation of the wound is necessary, it has to be kept in mind that the solutions' temperature must be according to patient's needs as solutions that are too cold or too warm may 
cause pain. External measures may be additionally helpful as well. Herbal cushions for the patient, essential oils, room odour neutralisers, or pleasant smelling substances - like a bowl of coffee powder in the room - may alleviate malodour.

\section{Local Care - Necrosis}

Tumour growth, reduced local blood supply, and loss of tissue viability lead to necrosis [1] (fig. 1). Necrotic tissue may have a disgusting appearance and is prone to be colonised by bacteria leading to discharge and putrid smell. If necroses are present, debridement (surgical, larval, enzymatic, or osmotic) may be appropriate. The decision to perform debridement at all - and if performed, which measures to use - has to be made balancing the possible benefit against the expected negative impact on the patient's comfort. If life expectancy is short and debridement carries the risk of enhancing profuse exudation, the strategy to leave the dead tissue in place and maintain a dry wound environment will serve the patient's needs better than invasive options [1]. Moreover, it should be kept in mind that irritation by rubbing or even cold air can cause pain and should be avoided. If irrigation of the wound is indicated, the solutions' temperature must be according to the patient's needs.

\section{Local Care - Bleeding}

The majority of fungating wounds will show minor, capillary bleeding on the surface (fig. 1). This may occur during or after a dressing change, or spontaneously. The tendency to bleed has to be taken into account when choosing the dressing material, for the most efficient management of bleeding is prevention. Alginates for example tend to evoke minor mechanical damages to the wound surface with subsequent haemorrhage upon removal. Non-fibrous materials that include silicone wound contact dressings may be useful [1]. Minor bleeding can be effectively contained by topically applied adrenaline (diluted to $1: 1,000$ ), but this drug should be used rather restrictively as adrenaline - a potent vasoactive agent - may aggravate tissue necrosis. Additionally, in more profuse capillary haemorrhage, haemostatic surgical sponges are helpful. Topical or systemic tranexamic acid may effectively promote haemostatis. In selected cases, radiotherapy is effective in reducing acute bleeding in ulcerating cancers [4].

Occasionally, ulcerating tumours erode major vessels and cause a massive and fatal external haemorrhage. These situations are highly distressing to patients, families, and professionals. If no haemostatic treatment options exist, advance planning (providing dark, blood-absorbing towels in the room, dark bed-linen to reduce the alarming red colour of blood in the case of massive bleeding), and information about the imminence of such an event are essential for all involved

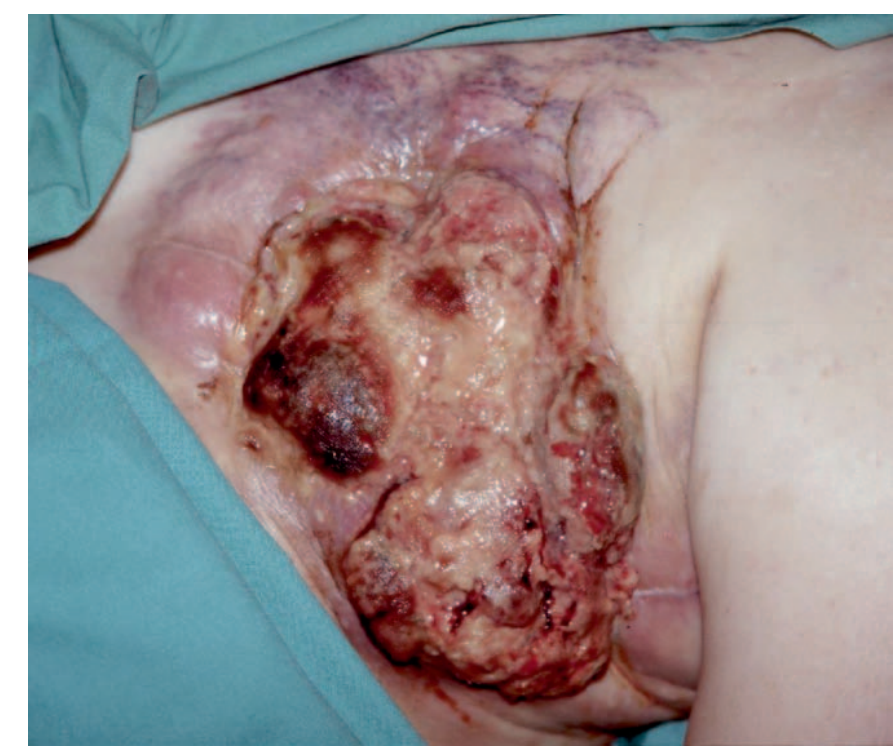

Fig. 1. Ulcerating tumour showing local bleeding and necrosis.

parties to be able to cope with the situation. The patient bleeding from a major vessel will die within minutes. Sedation is usually not necessary, as unconsciousness and consecutive death due to acute blood loss will occur faster than a drug would be able to act.

\section{Psychological, Social, and Spiritual Dimensions}

The visible signs of malignancy are often a particular psychological burden for the patient who experiences dissolution of his or her body integrity. This may precipitate depression and withdrawal from social activities. The penetration of the skin by the tumour implies that the disease is directly perceptible at all times. The disintegration of tissue can be interpreted by the patient as a symbol of her own physical decay: the perception of her own body changes radically. Parallel to the change in body appearance, other problems may arise such as pain or immobility. This stage of the illness can lead to significant emotional reactions, with the patient being repelled by herself and feeling ashamed in the presence of her partner, family members, or professional caregivers. The fear of being rejected by other people and the feeling of being unacceptable for them may result in social isolation.

Moreover, the female breast being visibly affected may cause problems within the patient's relationship, as the female breast is a part of the body that is closely related to tenderness and sexuality and often seen as a symbol of 'being a woman'. Both for the woman affected and for her partner this can lead to a dramatic change of roles within the relationship and to an end of its sexual aspect [5]. This in turn reinforces and promotes a feeling of worthlessness. 
Both the patients and the members of their families suffer from social isolation. In addition, feelings of guilt and shame arise - caused both by helplessness and powerlessness facing the disease and by one's own revulsion and distaste for the appearance and odour of the wound. These feelings of guilt along with the non-acceptance of one's own reactions to the wound can lead to a variety of reactions ranging from rejection of the sick partner to complete denial of the disease. Another effect is the inability to speak about the wound at all. On the other hand, family members may adopt a new role, being integrated into care. In taking an active role in the treatment process - for example by changing wound dressings family members may regain physical contact which gives reassurance to themselves and the patient to be able to cope with the situation. To integrate family members actively in the treatment process can reduce feelings of helplessness and exclusion. These integrative measures must however be considered very carefully in advance and then re-evaluated once implemented to monitor whether care is becoming too burdensome for the family members, which would aggravate the problems.

For patients with ulcerating tumours, health care professionals have to provide guidance and maximum safety. However, the health care professionals' own feelings play an active and very important role. Team members overcome the feelings of revulsion and distaste which often cannot be avoided when seeing and smelling wounds of this type and learn to cope with these reactions. In this context, unambiguous and sincere communication, a thorough and careful explanation of therapeutic strategies and goals, and professional management of wound-associated symptoms (pain, bleeding, infection, and malodour) are essential. Simply being aware of one's own anxiety and that of other people involved, facilitates a feeling of safety. Understanding the treatment measures and the direction the condition is developing in can reduce feelings of insecurity and anxiety.

\section{Conclusion}

In caring for patients with life-threatening diseases, the fundamental meaning of these situations for the patients, their relatives, and caregivers has to be kept in mind. Fungating wounds may cause not only physical harm but also social, psychological, and existential suffering. Therefore, their management is a challenge for the entire palliative care team. The aim of treatment is to alleviate the burden of the disease, to improve quality of life, and to maintain the feeling of dignity as far as possible. Apart from assessment, documentation, questions of care, wound dressing, and medical issues, social, psychological, and spiritual aspects have to also be addressed. Both up-to-date knowledge about techniques in wound care and multidisciplinary cooperation of nurse specialists, physicians, chaplains, psychologists, and others are required to meet this challenge.

\section{References}

1 Grocott P, Robinson V: Skin problems in palliative care - nursing aspects; in Hanks G, Cherny N, Christakis N, Kaasa S, Portenoy R (eds): The Oxford Textbook of Palliative Medicine, 4th ed. Oxford, Oxford University Press, 2010, pp. 961972.
2 World Health Organisation: Cancer Pain Relief, 2nd ed. Geneva, WHO, 1996.

3 Molan P: The evidence supporting the use of honey as a wound dressing. Int J Low Extrem Wounds 2006;5:40-54.
4 Sharma JB, Pushparaj M, Kumar S, Roy KK, Raina V, Malhotra N: Successful pregnancy outcome with 5-fluoruracil, epirubicin, cyclophosphamide therapy, and hemostatic radiotherapy with abdominal shielding for metastatic invasive intraductal breast carcinoma. Arch Gynecol Obstet 2009;279:415-417.

5 Lund-Nielsen B, Müller K, Adamsen L: Malignant wounds in women with breast cancer: feminine and sexual perspectives. J Clin Nurs 2005;14:56-64. 